\title{
Budget Allocation as a Multi-Agent System of Contextual \& Continuous Bandits
}

\author{
Benjamin Han \\ Lyft \\ San Francisco, California \\ bhan@lyft.com
}

\author{
Carl Arndt \\ Lyft \\ San Francisco, California \\ carndt@lyft.com
}

\begin{abstract}
Budget allocation for online advertising suffers from multiple complications, including significant delay between the initial ad impression to the call to action as well as cold-start prediction problems for ad campaigns with limited or no historical performance data. To address these issues, we introduce the Contextual Budgeting System (CBS), a budget allocation framework using a multi-agent system of contextual \& continuous Multi-Armed Bandits. Our proposed solution decomposes the problem into a convex optimization problem whose objective is drawn using Thompson Sampling. In order to efficiently deal with context and cold-start, we propose a transfer learning mechanism using supervised learning methods that augment simple parametric models.

We apply an implementation of this algorithm to all spending for new driver acquisition at Lyft and measure a $(22 \pm 10) \%$ improvement in the mean Cost Per user Acquisition (CPA) over a previous non-contextual model, based on Markov Chain MonteCarlo, generating tens of millions of dollars annually in efficiency improvements while also increasing total user acquisition.
\end{abstract}

\section{CCS CONCEPTS}

- Computing methodologies $\rightarrow$ Reinforcement learning; Multiagent reinforcement learning; Transfer learning; • Theory of computation $\rightarrow$ Bayesian analysis.

\section{KEYWORDS}

Reinforcement Learning, Advertising, Contextual Bandit, Continuous Multi-Armed Bandits, Thompson Sampling, Multi-Agent Learning, Transfer Learning, Portfolio Management

\section{ACM Reference Format:}

Benjamin Han and Carl Arndt. 2021. Budget Allocation as a Multi-Agent System of Contextual \& Continuous Bandits. In Proceedings of the 27th ACM SIGKDD Conference on Knowledge Discovery and Data Mining (KDD '21), August 14-18, 2021, Virtual Event, Singapore. ACM, New York, NY, USA, 9 pages. https://doi.org/10.1145/3447548.3467124

Permission to make digital or hard copies of all or part of this work for personal or classroom use is granted without fee provided that copies are not made or distributed for profit or commercial advantage and that copies bear this notice and the full citation on the first page. Copyrights for components of this work owned by others than ACM must be honored. Abstracting with credit is permitted. To copy otherwise, or republish, to post on servers or to redistribute to lists, requires prior specific permission and/or a fee. Request permissions from permissions@acm.org.

KDD '21, August 14-18, 2021, Virtual Event, Singapore

(C) 2021 Association for Computing Machinery.

ACM ISBN 978-1-4503-8332-5/21/08 . .\$15.00

https://doi.org/10.1145/3447548.3467124

\section{INTRODUCTION}

The explosion in the online advertising market has helped the growth of numerous firms over the last few decades. While traditional marketing channels were limited in their capacity to select personalized advertisements or measure user engagement, online paid media can target extremely narrow demographic groups and can be optimized in real-time. This granularity of control has significantly improved the return on investment of advertising and stimulated new fields of research devoted to advertising algorithms.

Lyft provides a taxi-network for its users by dispatching drivers to ride requests. Lyft invests considerable time and effort to balance driver supply and rider demand. Some levers to optimize the supply-demand balance have rapid responses (surge pricing mechanisms can suppress rider requests within minutes) while others have longer delays (driver notifications or incentives will not generate additional driver supply for hours). A slow but cost-efficient approach to manipulating supply and demand is user acquisition in the form of rider app installation and new driver application, respectively.

Our focus in this paper is a strategy to improve the cost-efficiency of driver acquisition through paid online advertising. Each prospective driver at Lyft has to pass a background check and other lengthy steps in the application process, creating a months-long delay between the initial ad impression to actively transporting passengers. There is also significant applicant churn, introducing uncertainty into whether or not the ad viewer will eventually become a working driver. An example driver ad can be seen in Figure 1.

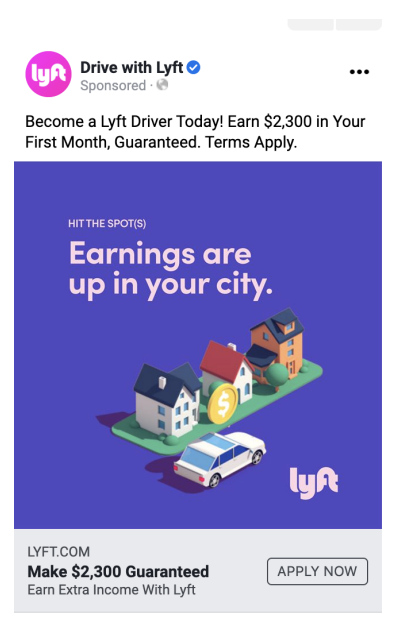

Figure 1: An example of ad creative and bonus incentive used by Lyft for driver acquisition 
The problem we face is generating a policy for allocating budgets across a set of given targeted ad campaigns that maximizes future rewards given budget/risk constraints, historical performance data, and contextual information. Regardless of the methodology, the solution has to explicitly deal with each of the following challenges:

- The response can be delayed over an unknown time horizon spanning months or potentially years.

- The response is influenced by unobserved external environmental factors such as prevailing ad prices or the ads of competitors.

- Spending across all available ad campaigns has to be jointly optimized to satisfy business constraints.

- New campaigns are introduced daily, creating significant cold-start problems.

Our proposed solution, the Contextual Budgeting System (CBS), frames this challenge as a constrained multi-agent reinforcement learning problem [35], where the observed rewards are generated by a set of surrogate predictions. Each agent in the system, i.e. the ads, is considered an independent contextual Multi-Armed Bandit $(M A B)$ $[4,22]$ with a continuous action space $[3,21]$, since at each point in time we can only observe the reward for that exact allocation of spend. The multi-agent aspect comes into play as the agents share a broader environment and common budget constraints. In order to make the problem more tractable and and improve the rate of learning, we propagate the observations from each agent using transfer learning [25]. In particular, a supervised regression model is trained across the full sample set. The knowledge encoded in this model is transferred to each agent by generating a set of pseudo-samples using the most-recent contextual features and a set of randomly-sampled proposal budgets. These pseudo-samples augment the observed samples used while learning each MAB. By assuming some parametric form of the reward as a function of the action, we use a parametrized model that results in a distribution of plausible reward functions for each agent. In order to explore each agent, we apply Thompson Sampling (TS) [27, 30] on a peragent basis and treat a randomly-sampled reward function as the true reward function while optimizing the budget allocation for the round. The budget problem is shown to be convex provided arbitrary convex constraints.

Our main contribution can be summarized as,

- Presenting a practical end-to-end system for budget allocation problems with delayed responses that is directly applicable to online advertisement budgeting

- A general algorithm for transferring common knowledge using supervised learning for multi-agent contextual multiarmed bandits

\subsection{Related Work}

Our budget allocation optimization strategy follows directly from [16] and is successfully applied by Criteo [15] and Netflix [23]. The core idea is the assumption that advertising campaigns yield diminishing marginal returns with increased budget allocation. However our contribution generalizes a typical budgetary constraint into arbitrary convex exploration constraints and we provide a simple long-term profitability constraint as a motivating example. While we have not seen surrogate-reward learning being directly applied to delayed responses for budgeting, the issue of missing samples for a probability-constrained budgeting is studied in [20].

In terms of the surrogate learning process, our approach is most similar to [12] except that they also attempt to consider causality. The Negative Binomial Delayed Feedback mode of [13] similarly tries to predict the conversion likelihood from the first click, however they use a parametric model that does not use contextual information (which is central to our model). An alternative is to directly apply a linear bandit with delayed rewards as in [31,36].

To our knowledge, the previous work with the most similar methodology to ours is [28], as they apply MABs and TS in their solution, though their coupling to the contextual variables is through a generalized linear model. Their algorithm is designed for placing advertisements given a budgetary constraint, however our framing differs by supporting a continuous action-space. The multi-agent approach of [19] is also similar in spirit, however they focus directly on the customer feedback loop in bidding, which is only feasible for advertising platforms and not for advertisers themselves. Other approaches $[2,10]$ have also studied the usage of MABs in online advertisement, though without attention to the budgeting problem.

Regarding multi-agent learning, TS is applied to multi-agent bandits with neighborhood structure in [32] and shown to converge, which bares some resemblance to our method where the transfer is based on contextual features in a global model. It has also been studied in the Contextual Bandit [7], though not sampled using TS. A transfer method has been proposed for multi-agent MABs in [5], but under the assumption that all MABs operate sequentially. This work also bears a large resemblance to [24] where the term Transferable Contextual Bandits is introduced. However they focus on online recommendations and transfer contextual information using a collaborative filtering method. They also show that this mitigates the cold-start problem (which is also a common motivation for our approach).

For continuous bandits (or for bandits with infinite arms), TS has been shown to converge with bounded regret in [26], which motivates our usage of it here. The extension of this work to Gaussian Process in [8] also bears some resemblance to our contextual setting. This problem also bears a large resemblance to Online Convex Optimization with Stochastic Bandit Feedback [1].

Going forward, our results motivate the development of large scale reinforcement learning approaches at Lyft, similar to those in production at other organizations, such as Facebook's Ax [14] and ReAgent/Horizon [17], and Google's Analytics Content Experiments [29] and REINFORCE [11]. A dedicated Bandit Platform for orchestrating adaptive field experiments, similar to those already in service at Facebook [6], has measured a $40 \%$ improvement to Lyft Pink membership subscription rates and has been applied to banner advertisements, messaging (email, SMS, push), the Lyft homepage, and pricing. We are also evaluating an approach to dispatching drivers using an MDP formulation defined by Didi [34] using a novel, online event-based Temporal Difference algorithm adopted from our winning entry at the 2020 KDD Cup (Reinforcement Learning Track) [18]. 


\section{BUDGET ALLOCATION}

The problem we consider is how to allocate spending to various ad campaigns to maximize utility under a set of constraints. In principle, utility can be anything measurable or possible to estimate, e.g. number of clicks, leads (viewers who may later convert), the number of drivers acquired, or the expected Lifetime Value (LTV) of new drivers. This problem is not unique to customer acquisition in online advertising, but exists anywhere one has a finite set of investment options and a delayed payout that is only observable after the action has been taken. We follow previous work on advertising budget optimization $[15,16,23]$ for an idealized setting with some minor modifications. Then, we extend this framing to the real-world, where forecasts are imprecise and actions have risk and uncertainty.

\subsection{Problem Formulation}

Consider a firm with a set of $N$ ad campaigns operating concurrently. Each round, for a given ad campaign $i$, an allocated spend $x_{i}$ yields a payout $y_{i}$. In particular we assume the payout is drawn from some distribution $\mathbb{E}\left[y_{i} \mid x_{i}\right]=f_{i}\left(x_{i}\right)$ s.t. $\mathbb{E}\left[\left(y_{i}\left(x_{i}\right)-f_{i}\left(x_{i}\right)\right)^{2} \mid x_{i}\right]<$ $\infty$ for $x_{i}, y_{i} \geq 0$. To simplify the problem, we assume that the set of payouts are mutually independent across the campaigns. However in reality this may not hold as campaigns can interact with one another due to competition in ad placements and cannibalization across campaigns/channels. We will refer to each function $f_{i}(x)$ as a payout function, which are assumed to satisfy Assumption 1.

Assumption 1. Assume that every payout function $f_{i}(x): \mathbb{R}_{+} \rightarrow$ $\mathbb{R}_{+}$satisfies:

(1) $f_{i}(x)$ is continuous and smooth to at least the second order.

(2) $f_{i}(x)$ is monotonically increasing (greater spend always yields greater utility), i.e. $f_{i}(x)^{\prime}>0$.

(3) $f_{i}(x)$ has a diminishing marginal impact, i.e. $f_{i}(x)^{\prime \prime}<0$.

In practice, we often observe that a successful ad campaign saturates and that increasing the budget allocation, $x$, no longer yields additional customers after the entire addressable audience has been reached (diminishing marginal returns). Furthermore, diminishing marginal returns ensures that campaigns with extraordinary returns have a finite allocation while allowing room for budget growth after confidence in the payout forecast grows with additional observations.

Given an oracle with perfect knowledge of each payout function, the target budgets $\hat{x}_{i} \geq 0$ can be found as the solution to

$$
\begin{array}{cl}
\underset{x \in \mathbb{R}_{+}}{\operatorname{maximize}} & \sum_{i=1}^{N} f_{i}\left(x_{i}\right) \\
\text { subject to } & H(x) \leq 0 \\
& G(x)=0
\end{array}
$$

Where $H(\cdot): \mathbb{R}_{+}^{N \times n} \rightarrow \mathbb{R}^{n}$ and $G(\cdot): \mathbb{R}_{+}^{N \times m} \rightarrow \mathbb{R}^{m}$ are assumed to be arbitrary convex function constraints. In the case of driver acquisition, these could correspond to budget constraints on a regional basis, efficiency constraints based on estimated LTV, or a single campaign constraint due to available audience saturation. Note here that Assumption 1 implies that $f(x)$ is concave; hence the above optimization program is convex [9].

\subsection{Marginal Efficiency Based Constraints}

Budgeted spend for each campaign is primary controlled by the marginal efficiency of the campaign. While [16] evaluates marginal efficiency based on the incremental payout per unit of spend, or $\frac{d f_{i}\left(x_{i}\right)}{d x_{i}}$, we choose to constrain allocations using a profitability constraint based on its inverse, or the Cost Per Incremental Acquisition (CPIA). For a given ad campaign, this metric describes, at the current spend level, the cost to acquire one additional unit of payout or the expected advertising cost for an additional driver. Provided target driver LTV estimates $c_{i}$, we form constraints as the following,

$$
\left[\frac{d f_{i}\left(x_{i}\right)}{d x_{i}}\right]^{-1}-c_{i} \leq 0
$$

In other words, budget allocations can be considered profitable when the marginal cost of acquisition does not exceed the estimated utility of each incremental driver acquisition.

Lemma 1. Under Assumption 1, the marginal efficiency constraints of form $\left[\frac{d f_{i}\left(x_{i}\right)}{d x_{i}}\right]^{-1}-c_{i} \leq 0$ are convex.

These constraints can thus be used in the budget optimization while preserving convexity. Note that in the special case of only having marginal efficiency constraints, the optimal budget allocation is trivially obtained as the solution to $\left[d f_{i}\left(x_{i}\right) / d x_{i}\right]^{-1}=c_{i}$, which we will apply in later sections.

\subsection{Challenges Arising from Uncertainty}

The previous sections provide a backbone for how to find optimal allocations satisfying the business constraints, conditional on full knowledge of the payout functions. However in practice, the main challenge is to generate reliable forecasts for these returns. In particular, we want to generate forecasts $\hat{f}_{i}(x)$ from the available historical information.

Before diving deeper into this, we highlight four challenges to estimating the payout functions:

(1) Dynamic context: An ad may compete in an ad marketplace day after day, but its payout function may change over time (seasonality, ad auction competitor behavior, or the available audience and their preferences could change).

(2) Extrapolation: An effective model must predict payouts for allocations outside the range of previously observed budgets.

(3) Cold start: We require stable payout predictions for new ads with limited or no historical data.

(4) Non-measurability: The true payout is only measurable in

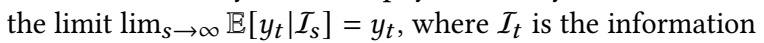
available at round $t$.

\section{TRANSFER LEARNING FROM CONTEXT}

While the previous section outlined how to allocate budgets in an ideal setting, here we address the issues outlined in Section 2.3. The aim of this section is to introduce our general algorithm while Section 4 goes into detail about the application to advertising used at Lyft.

Our Contextual Budgeting System (CBS) utilizes contextual features through a sequence of supervised learning problems and Bayesian methods to predict and explore the outcomes of each arm. 
Our system explores available actions by estimating the uncertainty of the payout distributions and samples from these distributions using TS. This cycle of predicting payouts, sampling from the prediction distributions, and performing constrained exploration repeats each round. The exploration process is worthwhile if it generates observations that improve the precision of future predictions.

An alternative to our approach could be creating an end-toend model for the entire process, naively training a deep neural network in supervised learning. The main shortcomings of this type of approach would be:

(1) Confidence: Our end model should be interpretable and have explicit uncertainty estimates. This helps gain confidence and explain exploration behavior to decision makers.

(2) Robustness: Our goal is not the payout function, but the budget allocation derived from these payouts. Hence it is crucial that the payout function is well behaved point-wise not just locally, but also globally.

\subsection{Contextual Budget System}

Expanding on our notation in Section 2, let's assume that we so far have allocations and observations for rounds $t=0, \ldots, T-1$ for each contextual MAB $i$ of the $N$ arms. For each round and $\mathrm{MAB}$ we also observe a vector of contextual features, $X_{i, t}$, and a delayed reward, $y_{i, t}$, which may be inaccurate or incomplete until much later. The outcome of each round is a new set of immediately available intermediate signals $Z_{i, t}$ from which we can forecast a future reward attributable to round $t, \hat{y}_{i, t}$ (though this forecast may have significant uncertainty). Our objective is to provide target budget allocations, $\hat{x}_{i, T}$, for each MAB agent that minimizes the regret,

$$
\sum_{i, t} y_{i, t}\left(x_{t}\right)-\max _{\hat{x}_{t} \in \mathcal{S}_{t} \forall t} \sum_{i, t} y_{i, t}\left(\hat{x}_{i, t}\right)
$$

where $S_{t}$ is the set of possible allocations from the constraints specified by (1).

Firstly, we rely on a surrogate rewards model, $h(y, Z)$, to generate an immediately available estimate for future rewards attributable to the actions of the previous rounds. From this model, we predict reward estimates $\hat{y}_{i, t}=h\left(y_{i, t}, Z_{i, t}\right)$ to replace the currently available observations $y_{i, t}$, which are known to be incomplete due to delayed rewards.

Then, we train a global model, $g(x, X)$, fitting $\hat{y}_{i, t}=g\left(x_{i, t}, X_{i, t}\right)$ from the entire data set of all observed contexts $X$, allocated spends $x$, and surrogate rewards $\hat{y}$. This model has no constraints or limitations and merely needs to provide accurate and unbiased pointestimates for the final return based on the context and budget allocation. Furthermore, this model can be applied to new contexts (including previously unobserved contexts, i.e. cold start).

Using an arbitrary distribution, we can draw a set of $k$ proposal budgets $\left\{\tilde{x}_{i, j}\right\}_{j=0}^{k}$ per bandit $i$ and generate a set of pseudo-samples $\left\{\tilde{x}_{i, j}, g\left(\tilde{x}_{i, j}, X_{i, T-1}\right)\right\}_{j=0}^{k}$. This data set augments the existing data set, $\left\{x_{i, t}, \hat{y}_{i, t}\right\}_{t=0}^{T-1}$. Using the augmented data set, we fit a new model, $f_{i}(x)$, which is distinct for each arm and is referred to as the local model. Each local model provides a distribution of plausible outcomes as a function of the target budget. By sampling from payout distributions, we get the final budget payout function, $\hat{f}_{i}(x)$, which can be plugged into (1) to yield the target allocation, $\hat{x}_{i, T}$.

In summary, we have decoupled the campaign portfolio into a multi-agent system of continuous, independent MAB agents that are explored using Thompson Sampling. The algorithm is summarized in Algorithm 1 and illustrated in Figure 2.

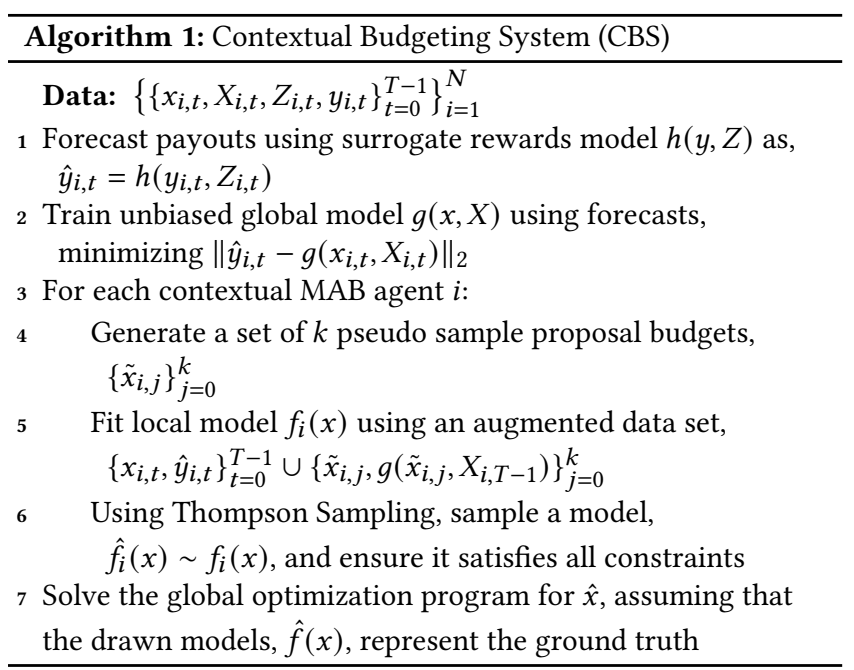

\subsection{Surrogate Learning a Delayed Reward}

The first step in our process is to estimate the payout attributable to each contextual MAB from the previously allocated budgets. Because of delayed rewards, the immediate measurement $y$ is incomplete and not a meaningful target for optimization. Instead, we rely on a slew of immediately available intermediate signals in a surrogate reward model, $h(y, Z)$, to generate a more reliable target, $\hat{y}$.

The intermediate signals $Z$ do not have to be disjoint from the contextual MAB features $X$, but they do include additional observations relevant to reward outcomes, such as user-specific information for every advertisement click or lead generated with high temporal precision. Some signals are measurable at the time of the spend (such as what type of device was used to access the ad), whereas other information is only observed later (such as driver applicant age, background check remarks, and vehicle ownership).

From this information, we train a non-linear model predicting the final payout $y_{i, t} \mid \mathcal{I}_{\infty}$. Any reasonable model should satisfy $\lim _{s \rightarrow \infty} h\left(y_{i, t} \mid \mathcal{I}_{s}, Z_{i, t}\right)=y_{i, t} \mid \mathcal{I}_{\infty}$, but it is also necessary to be unbiased on a shorter forecast horizon (even at the cost of high prediction variance). We utilize exact payout outcomes in the surrogate model when the outcome is finally observed and the delayed reward is finally realized or canceled. Our approach allows us to identify heterogeneity among the intermediate signals, emphasizing quality over quantity compared to a naive optimization over the measurements available immediately from the funnel (such as impressions, clicks, or leads). 


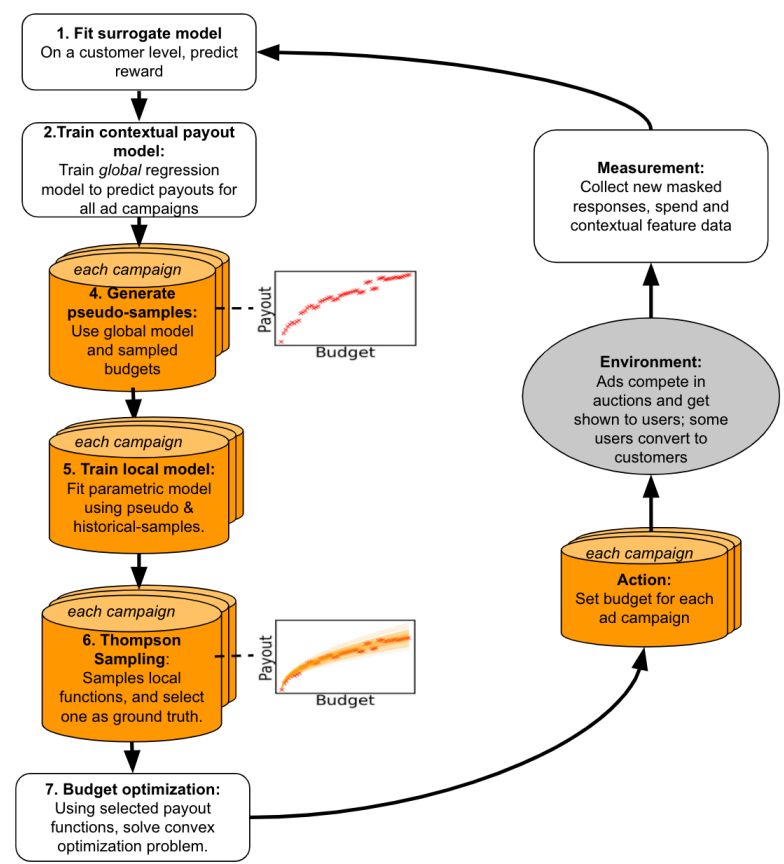

Figure 2: A schematic diagram of the Contextual Budgeting System with the numbering referring to Algorithm 1.

\subsection{Transfer Learning from Shared Context}

Our solution to estimate payouts is to train a global performance model $g(x, X)$ minimizing $\left\|\hat{y}_{t}-g\left(x_{t}, X_{t}\right)\right\|_{2}$, using all observations of historical budget allocations, contextual features, and predicted outcomes from the surrogate rewards model, $h(y, z)$.

In the driver acquisition setting, this data consists of the entire advertisement performance history, including deprecated or inactive ad campaigns. The model predicts payouts (acquired acquisition) using advertising features (including ad copy, sign-on bonus), audience features (including city, target audience, day of week), and the daily expenditure (including actual advertising costs and expected incentive bonus costs). Using a global regression model enables information-sharing among similar campaigns, compared to training separate models for each ad campaign. For example, an ad creative that was successful in New York is also likely to succeed in San Francisco.

A simple non-linear model, such as Random Forest or Gradient Boosted Trees, has excellent performance near-sample, when the budget does not vary significantly from recent observations. However most supervised approaches cannot reliably extrapolate; in the extreme, tree-based model predictions are clipped by both the minimum and maximum observed target values. Accordingly these predictions cannot be used to reliably forecast payouts when constraints are changed drastically, which is crucial for counterfactual analysis and planning. Similarly most naive supervised learning methods do not observe Assumption 1; in particular both aforementioned methods yield non-differentiable predictions.

\subsection{Transfer Process and the Local Model}

While non-linear machine learning models have evolved to the stage where they superbly outperform linear models, they still lack the interpretability and robustness a simple parametric model can offer.

To reliably forecast customer acquisition far-from-sample, where proposed budgets are distant in feature-space from the observed historical allocations, we fit a parametric model for the payout function for each ad campaign. The form guarantees computational efficiency and that the payout functions satisfy Assumption 1.

The key here is the transfer process, where a series of pseudosamples, $\left\{\tilde{x}_{i, j}, g\left(\tilde{x}_{i, j}, X_{i, T-1}\right)\right\}_{j=0}^{k}$, is generated from the contextual payout model and augments the historical samples, $\left\{x_{i, t}, \hat{y}_{i, t}\right\}_{t=0}^{T-1}$. In order to generate the pseudo-samples, we apply a simple parametric form that samples allocations that are not only plausible actions, but are also near-sample, where model performance is best. In particular, the number of generated pseudo-samples can greatly benefit payout predictions for bandits with limited or no historical samples (i.e. cold start) as measured in Section 5.

A crucial requirement is that the model generates a distribution of payouts, rather than just a mean estimate. This allows us to estimate model uncertainty, including the confidence intervals of proposed budgets. More importantly, it also dictates the explorationexploitation tradeoff, as we otherwise may incur regret by only exploiting actions that were immediately successful at launch.

\section{LYFT REALIZATION OF META ALGORITHM}

While Section 3.1 outlines a general framework for how to deal with contextual features and uncertainty, this section dives into the details of Lyft's advertising budget allocation process for user acquisition.

\subsection{Surrogate \& Global Models}

The user acquisition funnel has significantly delayed rewards from the first ad impression to active usage. This issue is especially severe for the driver acquisition funnel, which can take months before the successful applicant is eligible to transport passengers. To mitigate the uncertainty of these delayed rewards, gradient-boosted models are trained to forecast the acquisition likelihood of users at fixed time-horizons attributable to each ad campaign in a surrogate rewards model. Intermediate signals $Z$ includes applicant features, such as device type, device, operating system, or location. From these models, a logistic regression is used to smoothly interpolate the forecasts between each time-horizon.

To generate reasonable outcome estimates for data augmentation, we also train a new global model using a Random Forest in each round. For each ad campaign, the global model generates pseudosamples from a series of linearly-spaced proposal budgets chosen near the observed history where the models interpolates reliably. The global model predictions are combined with the historical 
budgets and surrogate rewards to generate local models for each ad campaign.

\subsection{Local Models}

For each campaign, we fit a simple power-law of form $y=\kappa_{1} x^{\kappa_{2}}$ constrained with $\kappa_{1}>0$ and $\kappa_{2} \in(0,1)$. To make this more tractable, we apply a $\log$ transform $\log (y)=\log \left(\kappa_{1}\right)+\kappa_{2} \log (x)^{1}$ and do the fitting in log-space. Figure 3 shows example results of fitting this model using linear-regression from the augmented data set.

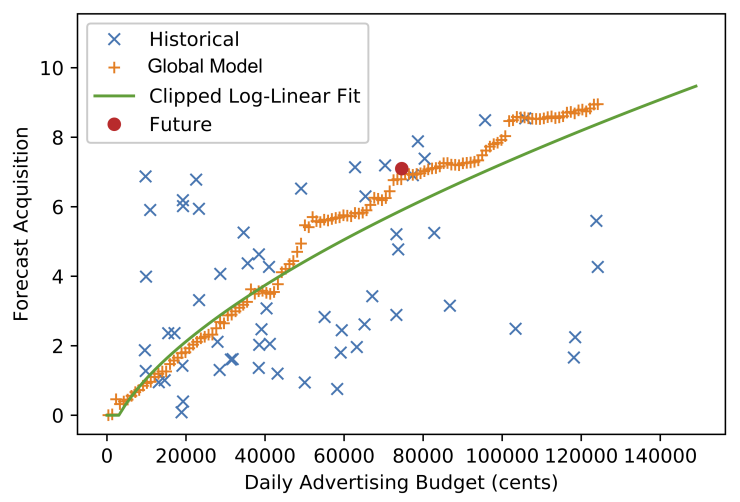

Figure 3: High variance historical observations are augmented by low-variance point predictions generated by a global model, $g\left(x, X_{T-1}\right)$.

4.2.1 Bayesian Linear Regression. While linear regression generates mean parameter weights, we chose to take a full Bayesian approach to include prior beliefs. This is particularly helpful for robustness to prevent extreme budget outliers from high-variance or sparse observation periods. Hence we instead rely on Bayesian Linear Regression to provide covariance estimates for Gaussiandistributed weights. From design matrix $X$ (containing historical budgets), historical acquisitions $\boldsymbol{y}$, prior inverse-gamma hyperparameters $\left(a_{0}, b_{0}\right)$, prior precision matrix $\Lambda_{0}$, and prior mean weights $\boldsymbol{\mu}_{0}$, we obtain posterior mean weights $\boldsymbol{\mu}$ and posterior precision matrix $\Lambda$ :

$$
\begin{aligned}
& \boldsymbol{\mu}=\left(\boldsymbol{X}^{T} \boldsymbol{X}+\Lambda_{0}\right)^{-1}\left(\Lambda_{0} \boldsymbol{\mu}_{0}+\boldsymbol{X}^{T} \boldsymbol{y}\right) \\
& \boldsymbol{\Lambda}=\boldsymbol{X}^{T} \boldsymbol{X}+\Lambda_{0}
\end{aligned}
$$

In this framing, the vector $\boldsymbol{\mu}=\left(\kappa_{1}, \kappa_{2}\right)$ gives an estimate of the shape and normalization of the power-law fit. The covariance can be evaluated from normal-inverse gamma parameters $a, b$ as follows [33],

$$
\begin{aligned}
\Sigma & =\frac{b}{a-1} \Lambda^{-1} \\
a & =a_{0}+|\boldsymbol{y}| / 2 \\
b & =b_{0}+\frac{1}{2}\left(\boldsymbol{y}^{T} \boldsymbol{y}+\boldsymbol{\mu}_{0}^{T} \Lambda_{0} \boldsymbol{\mu}_{0}+\boldsymbol{\mu}^{T} \boldsymbol{\Lambda} \boldsymbol{\mu}\right)
\end{aligned}
$$

${ }^{1}$ In production, we fit $\log (y+1)=\kappa_{1}+\kappa_{2} \log (x+1)$ to support zero values, but refer to the simpler form above for brevity without significant algorithmic changes.
Thus we obtain an estimate of the power-law payout curve distribution, with an uncertainty measure provided by the parameter covariance.

\subsection{Exploring the Budget Action-Space}

Because the Bayesian Linear Model provides an explicit parameter distribution $\left(\kappa_{1}, \kappa_{2}\right) \sim \mathcal{N}(\boldsymbol{\mu}, \Sigma)$, we can sample payout functions $f(x)=\kappa_{1} x^{\kappa_{2}}$. It is important to note that these distributions are skewed due to the inclusion of the pseudo-samples; in particular the covariance is skewed downwards as the pseudo-samples tend to have a lower variance than the historical samples. Figure 4 shows an example set of curves sampled from the posterior distribution of the Bayesian Linear Regression model in comparison with extremely noisy historical samples.

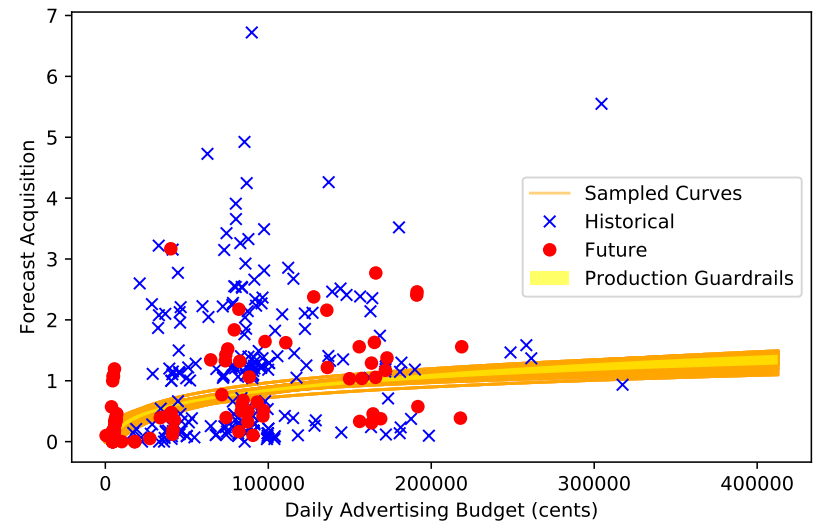

Figure 4: The payout curve distribution variance facilitates budget allocation exploration using Thompson Sampling. The curve distribution has lower variance due to data augmentation from pseudo-samples.

Using TS, we randomly choose one curve $\hat{f}_{i}(x)$ from the set of curves. This is a simple way to manage the explore-exploit tradeoffs based on uncertainty. This is particularly important for new campaigns, as they may otherwise be deemed inefficient and culled too quickly if initial performance is poor.

The selected models $\hat{f}_{i}(x)$ are then fed into Equation (1). We observe in Figure 5 how one campaign's budget allocation grows as a function of the target CPIA for the city, $c_{i}$. In Figures 4 and 5, we include guardrails (generated by quantile constraints) in production that limit budget distribution extremes.

The system exhibits greater budget exploration if an ad campaign has substantial performance variance by sampling curves from broader parameter distributions. Over time, the system accumulates a larger and more diverse volume of data than that of a pure-exploitation model, improving forecast accuracy in the long term as measured in Section 5. From a practical perspective, we can interpret the parameters as corresponding to efficiency $\left(\kappa_{1}\right)$ and addressable audience capacity $\left(\kappa_{2}\right)$ while communicating changes to marketing/operations teams. 


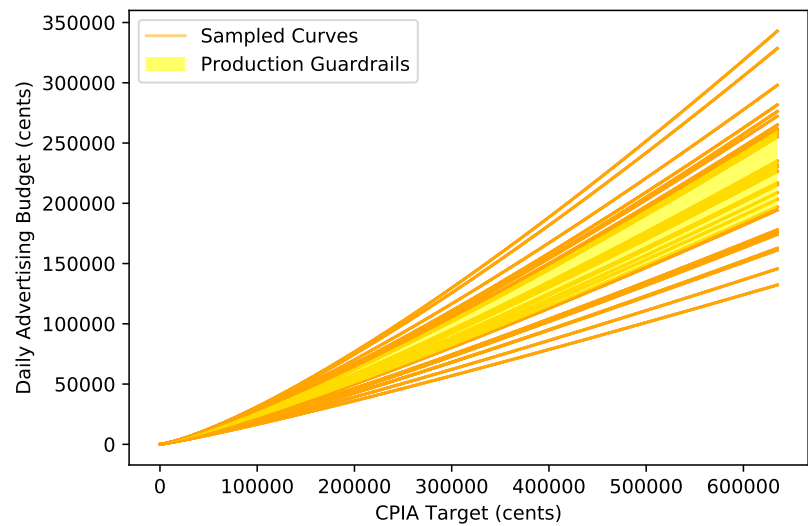

Figure 5: The allocated budget curve distribution grows exponentially as a function of the target CPIA allowance. CPIA is provided externally for each city based on forecasts for future driver supply, rider demand, and driver utilization.

\section{RESULTS}

We performed offline backtest using historical data to measure the payout prediction accuracy of various model architectures (Section 5.2), independent of the exploration policy. After selecting the Random Forest + Bayesian Linear Regression (RF_BLR) model, we compared CBS with our previous system (Section 5.1) in a regionsplit A/B test, measuring significant gains in both predictive power and cost efficiency (Section 5.3) after 7 weeks of on-policy control over two 3-week measurement periods. CBS is now responsible for allocating hundreds of millions of dollars annually.

\subsection{Baseline}

Our previous system was based on a parametric model (similar to the local model described in Section 4.2), however it did not incorporate a global model or any transfer learning. Instead, the system fit an independent model to the historical payouts data for each ad campaign. The system used a hierarchical model that required Markov Chain Monte Carlo sampling. We refer to this baseline model as the MCMC model below.

Roughly, MCMC is a method for estimating the best-fit distribution of the parameters in a model by sampling points in the parameter space and accepting or rejecting them based on their consistency with observations. The baseline implementation applied a similar form, $y=\kappa_{1} x^{\kappa_{2}}+\epsilon$, assuming Gaussian error, $\epsilon \sim \mathcal{N}\left(\mu_{\epsilon}, \sigma_{\epsilon}^{2}\right)$ and a $\Gamma$ distributed prior for $\kappa_{1}$ and a Beta distributed prior for $\kappa_{2}$.

The MCMC sampling was computationally expensive and prone to poor system performance, often leading to system failure (see Section 6.4).

\subsection{Offline Evaluation}

We compared the accuracy of payout predictions from several different regression models using historical data. We tested the previous implementation using MCMC, Least Squares Regression (LSR), Bayesian Linear Regression (BLR), Random Forest (RF), RF-augmented Least Squares Regression (RF_LSR), RF-augmented
Table 1: Offline Model Error (all advertisements)

\begin{tabular}{lccccc}
\hline Approach & Bias & MAE & MSE & Curve & Uncertainty \\
\hline MCMC & 0.1575 & 0.2144 & 0.5881 & $\checkmark$ & $\checkmark$ \\
LSR & -0.6384 & 0.1095 & 0.4242 & $\checkmark$ & $\boldsymbol{x}$ \\
BLR & -0.1029 & 0.1565 & 0.4024 & $\checkmark$ & $\checkmark$ \\
RF & $\mathbf{- 0 . 0 3 4 0}$ & $\mathbf{0 . 0 7 8 8}$ & $\mathbf{0 . 0 6 5 6}$ & $\boldsymbol{x}$ & $\boldsymbol{x}$ \\
RF_LSR & $\mathbf{- 0 . 0 3 6 4}$ & $\mathbf{0 . 0 8 9 4}$ & $\mathbf{0 . 0 8 7 1}$ & $\checkmark$ & $\boldsymbol{X}$ \\
RF_BLR & $\mathbf{- 0 . 0 3 4 9}$ & $\mathbf{0 . 0 9 3 7}$ & $\mathbf{0 . 0 9 4 6}$ & $\checkmark$ & $\checkmark$ \\
LGBM & -0.0764 & 0.1894 & 0.3463 & $\boldsymbol{x}$ & $\boldsymbol{x}$ \\
\hline
\end{tabular}

Table 2: Offline Model Error (cold start, less than 7 day data)

\begin{tabular}{lccccc}
\hline Approach & Bias & MAE & MSE & Curve & Uncertainty \\
\hline MCMC & 0.0842 & 0.123 & 0.0372 & $\checkmark$ & $\checkmark$ \\
LSR & 0.0364 & 0.1118 & 2.1390 & $\checkmark$ & $\boldsymbol{x}$ \\
BLR & $\mathbf{- 0 . 0 0 4 5}$ & $\mathbf{0 . 0 5 8 6}$ & $\mathbf{0 . 0 2 9 0}$ & $\checkmark$ & $\checkmark$ \\
RF & 0.0179 & 0.0714 & 0.0421 & $\boldsymbol{x}$ & $\boldsymbol{x}$ \\
RF_LSR & $\mathbf{0 . 0 0 8 7}$ & $\mathbf{0 . 0 6 6 8}$ & $\mathbf{0 . 0 2 6 4}$ & $\checkmark$ & $\boldsymbol{x}$ \\
RF_BLR & $\mathbf{0 . 0 0 8 1}$ & $\mathbf{0 . 0 6 8 2}$ & $\mathbf{0 . 0 2 5 7}$ & $\checkmark$ & $\checkmark$ \\
LGBM & 0.0366 & 0.0987 & $\mathbf{0 . 0 2 7 5}$ & $\boldsymbol{x}$ & $\boldsymbol{x}$ \\
\hline
\end{tabular}

Table 3: Online System Error (all advertisements)

\begin{tabular}{ccc}
\hline Approach & MAE & MSE \\
\hline MCMC & $0.1320 \pm 0.0207$ & $0.0716 \pm 0.0467$ \\
CBS & $\mathbf{0 . 0 7 3 2} \pm \mathbf{0 . 0 1 2 2}$ & $\mathbf{0 . 0 2 8 0} \pm \mathbf{0 . 0 1 3 2}$ \\
\hline
\end{tabular}

Bayesian Linear Regression (RF_BLR), and LightGBM (LGBM). We used training data with observations from January 1, 2019 April 13, 2019 and tested on data spanning April 14 - 27, 2019.

RF has the lowest error in Table 1, however the model is not differentiable and cannot be applied directly to our maximization strategy. This limitation also holds for LGBM. And while the combined RF_LSR approach yields the best curve-fit, neither it nor LSR provides uncertainty measures for exploration in Thompson Sampling.

We also examined the subgroup of campaigns with less than 7 days of training data to evaluate cold-start in Table 2. New campaigns are typically allocated more conservative budgets from priors and initially generate fewer customer acquisitions until demonstrating cost-efficient returns. This presents as lower absolute values in the unscaled error metrics shown in Table 2 than in Table 1. Regardless, RF_BLR yields the lowest Mean Squared Error (MSE) among strategies meeting our optimization program criteria outlined in Assumption 1. RF was deployed as the global model and the augmented RF_BLR was used in the local models in online experimentation.

\subsection{Online Experiments}

We measured our proposed system, $\mathbf{C B S}$ in a region-split A/B test in seven major cities. We launched CBS in four cities over 3 weeks while measuring three additional cities using the existing MCMC 
Table 4: Experiment Results

\begin{tabular}{lcc}
\hline City & CPA \% Improvement & Std Error \\
\hline Boston & 37.08 & 24.02 \\
Chicago & 32.56 & 57.23 \\
Denver & -0.01 (worse) & 11.95 \\
Philadelphia & 15.57 & 20.88 \\
Pittsburgh & 79.03 & 10.73 \\
San Francisco & 3.09 & 9.93 \\
Washington, DC & 9.92 & 23.50 \\
\hline
\end{tabular}

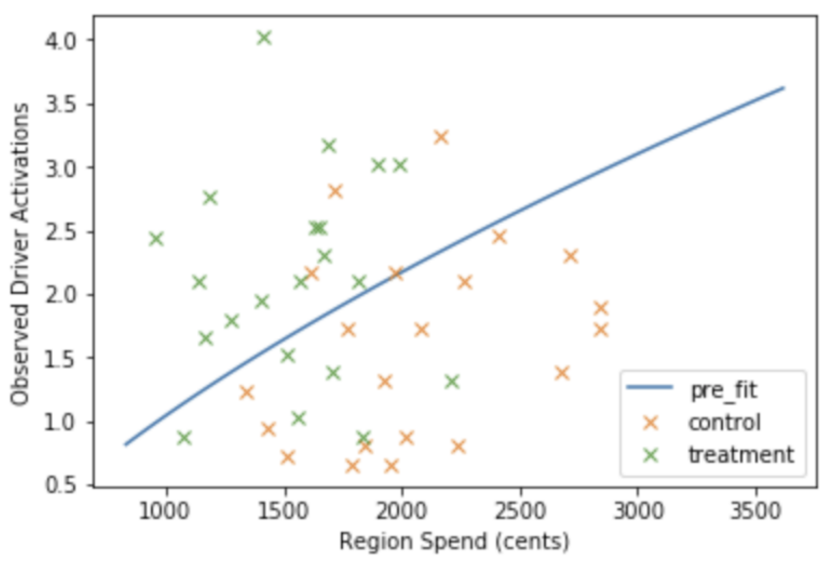

Figure 6: Daily driver acquisition using CBS (treatment) yields more total drivers at lower expense than the baseline approach using MCMC (control). The pre-fit curve is fit to measured returns prior to experiment launch.

approach as the control policy. After, we swapped control and treatment cities and continued experimentation for three additional weeks to evaluate the treatment effect within each city. During the control/treatment swap, we delayed measurement one week to mitigate experimental interference due to ad viewers who had been exposed to the previous strategy. We ensured the CPIA targets for test cities were held constant for the duration of the experiment.

In the online error evaluation, both systems are only responsible for prediction accuracy on a 1-day horizon (instead of up to 2-weeks as in the offline evaluation). This presents as lower absolute error in Table 3 than in Table 1. Our proposed system, CBS, dominated the baseline approach across error and business metrics.

Because it is difficult to measure CPIA or Marginal Return on Investment directly [15], we instead measure the mean Cost Per Acquisition (CPA). From pre-experiment measurements, we use Ordinary Least Squares to fit a curve $p$, providing an estimated baseline performance function defined for all budget values $x$, including for unobserved budgets. We consider the daily experimental spend $x$ and driver acquisition payout $y$ as a ratio $y / p(x)$. We then evaluate the improvement of treatment over control as a ratio. Figure 6 illustrates this measurement for one region. Controlling for location in Table 4, we measure an overall improvement in CPA of
(21.8 \pm 10.3$) \%$ between treatment and control. Similarly, we measure a $(21.5 \pm 13.1) \% \mathrm{CPA}$ improvement while controlling for time. These improvements correspond to tens of millions of dollars in savings for 2019. Shortly after the conclusion of this experiment, CBS was deployed to all regions.

\section{PRACTICAL CONSIDERATIONS}

The current production system retrains the model on a daily basis and generates a budget for the following day using a batch job system. As upstream data pipelines have non-trivial failure rates, various distribution checks have been added to improve the robustness, favoring stale model parameters from the last-good model over more recent, but potentially invalid training samples. Because TS samples actions stochastically, the system continues to explore new actions, even when the model parameters are unchanged and stale. As previously mentioned, exploration is restricted by guardrails, using the middle quartiles of the parameter distributions.

\subsection{Recency Weighting}

For training the global model, we use a weighted $L_{2}$-penalty based on time proximity in order to quickly adapt to changing environmental factors. In particular we used an exponential penalty with a half-life varying between two weeks to a month.

\subsection{Local Model Regularization}

The payout functions of the form $\hat{f}(x)=\hat{\kappa}_{1}(x+1)^{\hat{\kappa}_{2}}-1$ satisfy Assumption 1 if and only if $\hat{\kappa}_{1} \geq 0$ and $0 \leq \hat{\kappa}_{2}<1$. However, it is possible that values are sampled outside of this regime, in particular for cases where the number of observations is very small. To resolve this, we iteratively increase the diagonal element of the prior precision matrix $\Lambda_{0}$ corresponding to the relevant parameter until the assumptions are satisfied. In Thompson Sampling, we also reject any sample that falls outside of the desired range.

\subsection{Hyperparameter Optimization}

Before manipulating real budgets, we conducted extensive hyperparameter optimization using multi-dimensional random searches in both offline and online off-policy settings. We searched scikitlearn Random Forest hyperparameters n_estimator $\in[10,512]$ and max_features $\in\{$ auto, $\log 2,0.1,0.25,0.5\}$ and Bayesian Linear Regression hyperparameters for the number of augmented data points $\in[128,512]$ and prior precision $\Lambda_{\kappa_{2}} \in[0,49]$. The number of augmented data points is an especially sensitive hyperparameter because it correlates with the posterior precision of the parameter distributions generated by Bayesian Linear Regression.

\subsection{Computational Advantages of our System}

In addition to improving the spend efficiency, the deployment of CBS reduced the necessary infrastructure from 15 to 1 AWS r5a.8xlarge instances. It also improved reliability by eliminating the possibility of chain failure in MCMC due to poorly fitting priors and failure to find model parameters within the allotted time. Deploying to CBS increased the Mean Time Between Failures from 2.04 to 21.0 days in the 100 most recent execution attempts. 


\section{DISCUSSION AND CONCLUSION}

In this paper, we present a general framework for applying transfer learning to a Contextual Budgeting System (CBS) with support for arbitrary constraints. Our system attempts to maximize total driver acquisition by allocating campaign budgets such that the mean cost of acquisition is profitable based on forecasted driver supply and rider demand. We outline Lyft's user acquisition implementation based on Random Forest and Bayesian Linear Regression that generates payout function distributions and explores diverse observations for future exploitation. In online experiments, we measure a $(22 \pm 10) \%$ improvement in the mean Cost Per Acquisition over the existing Markov Chain Monte Carlo method while controlling for location and report similar results while controlling for time.

\section{ACKNOWLEDGMENTS}

The author would like to thank Jared Gabor, Jared Bauman, Dongwei Cao, William Borges, Carolyn Conway, Antonio Luna, Jack van Ryswyck, Xing Xing, Alejandro Veen, Marisa Wong, Suma Snehalatha, Linda Dreyer, Don Dini, Di Wu, Sumeet Kumar, Hyungjun Lee, Michael Rotkowitz, Ilan Lobel, Gordon Rios, Patrick McGrath, Nir Even-Chen, David Fishman, Tianqi Wang, Hannah Young, Michael Yoshizawa, Lei Tang, Sara Smoot, and Craig Martell for their support on this project and paper.

\section{A PROOF OF LEMMA 1}

The constraint $\left[\frac{d f_{i}\left(x_{i}\right)}{d x_{i}}\right]^{-1}-c_{i} \leq 0$ can be rewritten as,

$$
\frac{d f_{i}\left(x_{i}\right)}{d x_{i}} \geq c_{i}^{-1}
$$

Note that the convexity assumption in Assumption 1 guarantees the existence of a unique solution to $\frac{d f_{i}\left(x_{i}\right)}{d x_{i}}=c_{i}^{-1}$ under the condition that a solution exists. In these cases we can define this solution as $\tilde{x}_{i}\left(c_{i}\right)$, and since convexity implies that the derivative is monotone it follows that we can reformulate (4) as $x_{i}-\tilde{x}_{i}\left(c_{i}\right) \leq 0$. If there are no solutions then continuity guarantees that either all or no values will satisfy (4), which proves the claim.

\section{REFERENCES}

[1] Alekh Agarwal, Dean P Foster, Daniel Hsu, Sham M Kakade, and Alexander Rakhlin. 2013. Stochastic convex optimization with bandit feedback. SIAM fournal on Optimization 23, 1 (2013), 213-240.

[2] Deepak Agarwal, Bee chung Chen, Pradheep Elango, Nitin Motgi, Seung taek Park, Raghu Ramakrishnan, Scott Roy, and Joe Zachariah. 2009. Online Models for Content Optimization. In Advances in Neural Information Processing Systems 21. $17-24$.

[3] Rajeev Agrawal. 1995. The continuum-armed bandit problem. SIAM journal on control and optimization 33, 6 (1995), 1926-1951.

[4] Peter Auer. 2002. Using confidence bounds for exploitationexploration trade-offs. fournal of Machine Learning Research 3 (2002), 397-422.

[5] Mohammad Gheshlaghi Azar, Alessandro Lazaric, and Emma Brunskill. 2013. Sequential transfer in multi-armed bandit with finite set of models. arXiv preprint arXiv:1307.6887 (2013).

[6] Eytan Bakshy, Lili Dworkin, Brian Karrer, Konstantin Kashin, Benjamin Letham, Ashwin Murthy, and Sonia Singh. 2018. AE : A domain-agnostic platform for adaptive experimentation.

[7] Santiago Balseiro, Negin Golrezaei, Mohammad Mahdian, Vahab Mirrokni, and Jon Schneider. 2019. Contextual Bandits with Cross-Learning. In Advances in Neural Information Processing Systems. 9679-9688.

[8] Kinjal Basu and Souvik Ghosh. 2017. Adaptive Rate of Convergence of Thompson Sampling for Gaussian Process Optimization. arXiv e-prints (2017), arXiv-1705.

[9] Stephen Boyd, Stephen P Boyd, and Lieven Vandenberghe. 2004. Convex optimization. Cambridge university press.
[10] Deepayan Chakrabarti, Ravi Kumar, Filip Radlinski, and Eli Upfal. 2009. Mortal Multi-Armed Bandits. Advances in Neural Information Processing Systems 21 (2009), 273-280.

[11] Minmin Chen, Alex Beutel, Paul Covington, Sagar Jain, Francois Belletti, and Ed Chi. 2018. Top-K Off-Policy Correction for a REINFORCE Recommender System.

[12] Lu Cheng, Ruocheng Guo, and Huan Liu. 2020. Long-Term Effect Estimation with Surrogate Representation. arXiv preprint arXiv:2008.08236 (2020).

[13] Youngmin Choi, Mugeun Kwon, Younjin Park, Jinsoo Oh, and Suyoung Kim. 2020. Delayed Feedback Model with Negative Binomial Regression for Multiple Conversions.

[14] Drew Dimmery, Eytan Bakshy, and Jasjeet Sekhon. 2019. Shrinkage Estimators in Online Experiments. Proceedings of the 25th ACM SIGKDD International Conference on Knowledge Discovery \& Data Mining - KDD '19 (2019).

[15] Vincenzo D'Elia. 2019. On the causality of advertising. http://papers.adkdd. org/2019/invited-talks/slides-adkdd19-delia-causality.pdf [Online; accessed 10December-2019]

[16] Paul W. Farris, Dominique M. Hanssens, James D. Lenskold, and David J. Reibstein. 2015. Marketing return on investment: Seeking clarity for concept and measurement. Applied Marketing Analytics 1 (apr 2015), 267-282.

[17] Jason Gauci, Edoardo Conti, Yitao Liang, Kittipat Virochsiri, Yuchen He, Zachary Kaden, Vivek Narayanan, Xiaohui Ye, Zhengxing Chen, and Scott Fujimoto. 2018. Horizon: Facebook's Open Source Applied Reinforcement Learning Platform.

[18] Benjamin Han. 2020. Reinforcement Learning: Mobility on Demand. https://github.com/laxatives/rl\#acm-sigkdd-cup-2020-learning-to-dispatchand-reposition.

[19] Junqi Jin, Chengru Song, Han Li, Kun Gai, Jun Wang, and Weinan Zhang. 2018. Real-Time Bidding with Multi-Agent Reinforcement Learning in Display Advertising. Proceedings of the 27th ACM International Conference on Information and Knowledge Management - CIKM '18 (2018).

[20] Xiao Jin, Yichi Shen, Haobin Li, Loo Hay Lee, Ek Peng Chew, and Christine A Shoemaker. 2020. Combining Adaptive Budget Allocation with Surrogate Methodology in Solving Continuous Scenario-based Simulation Optimization. In 2020 IEEE 16th International Conference on Automation Science and Engineering (CASE). IEEE, 1509-1513.

[21] Akshay Krishnamurthy, John Langford, Aleksandrs Slivkins, and Chicheng Zhang. 2019. Contextual bandits with continuous actions: Smoothing, zooming, and adapting. In Conference on Learning Theory. PMLR, 2025-2027.

[22] John Langford and Tong Zhang. 2008. The Epoch-Greedy Algorithm for Contextual Multi-armed Bandits. In Advances in Neural Information Processing Systems 20. 817-824.

[23] Randall A. Lewis and Jeffrey Wong. 2018. Incrementality Bidding \& Attribution.

[24] Bo Liu, Ying Wei, Yu Zhang, Zhixian Yan, and Qiang Yang. 2018. Transferable contextual bandit for cross-domain recommendation. In Proceedings of the AAAI Conference on Artificial Intelligence, Vol. 32.

[25] Sinno Jialin Pan and Qiang Yang. 2009. A survey on transfer learning. IEEE Transactions on knowledge and data engineering 22, 10 (2009), 1345-1359.

[26] Daniel Russo and Benjamin Van Roy. 2014. Learning to optimize via posterior sampling. Mathematics of Operations Research 39, 4 (2014), 1221-1243.

[27] Daniel J. Russo, Benjamin Van Roy, Abbas Kazerouni, Ian Osband, and Zheng Wen. 2018. A Tutorial on Thompson Sampling. Found. Trends Mach. Learn. 11, 1 (July 2018), 1-96.

[28] Eric M. Schwartz, Eric T. Bradlow, and Peter S. Fader. 2017. Customer Acquisition via Display Advertising Using Multi-Armed Bandit Experiments. INFORMS Marketing Science (2017).

[29] Steven L. Scott. 2010. A modern Bayesian look at the multi-armed bandit. Applied Stochastic Models in Business and Industry 26, 6 (2010), 639-658.

[30] William R. Thompson. 1933. On the Likelihood that One Unknown Probability Exceeds Another in View of the Evidence of Two Samples. Biometrika 25, 3/4 (1933), 285-294.

[31] Claire Vernade, Alexandra Carpentier, Tor Lattimore, Giovanni Zappella, Beyza Ermis, and Michael Brueckner. 2020. Linear bandits with stochastic delayed feedback. In International Conference on Machine Learning. PMLR, 9712-9721.

[32] Timothy Verstraeten, Eugenio Bargiacchi, Pieter JK Libin, Jan Helsen, Diederik M Roijers, and Ann Nowé. 2020. Multi-agent Thompson sampling for bandit applications with sparse neighbourhood structures. Scientific reports 10, 1 (2020), $1-13$.

[33] Gero Walter and Thomas Augustin. 2010. Bayesian Linear Regression - Different Conjugate Models and Their (In)Sensitivity to Prior-Data Conflict. 59-78.

[34] Zhe Xu, Zhixin Li, Oingwen Guan, Dingshui Zhang, Oiang Li, Junxiao Nan, Chunyang Liu, Wei Bian, and Jieping Ye. 2018. Large-Scale Order Dispatch in On-Demand Ride-Hailing Platforms: A Learning and Planning Approach. In Proceedings of the 24th ACM SIGKDD International Conference on Knowledge Discovery \& Data Mining. 905-913.

[35] Kaiqing Zhang, Zhuoran Yang, Han Liu, Tong Zhang, and Tamer Basar. 2018. Fully decentralized multi-agent reinforcement learning with networked agents. In International Conference on Machine Learning. PMLR, 5872-5881.

[36] Zhengyuan Zhou, Renyuan Xu, and Jose Blanchet. 2019. Learning in generalized linear contextual bandits with stochastic delays. (2019). 